\title{
Stereo Vision System \& 3D TV Program Production
}

\author{
Zhenlong Zhang, Taozheng Zhang, Jianping Chai \\ Information Engineering School \\ Communication University of China, China \\ Beijing, China \\ E-mail: 1072547168@qq.com, zhangtaozheng@cuc.edu.cn,jp_chai@cuc.edu.cn
}

\begin{abstract}
Stereo vision is a strong-crossed subject, and it has been one of the most hot topics in the study of computer. In more than 40 years of development, it has formed its own method and theory. This paper systematically reviews the present situation of stereo vision research, briefly introduces stereo vision system and 3D TV program production, and analyzes the error controlling through the production.
\end{abstract}

Keywords- Stereo Vision; Stereo Vision System; 3D TV program production

\section{INTRODUCTION}

Stereo vision is an important and cross subject, rising in the mid - 1960s, and its main studies is how to use imaging technology (multiple images) to get the distance (depth) of the object in the scene from many images. The basic method of $3 \mathrm{D}$ is to use two or more perspectives to observe the same target object. From this method, we can obtain a set of images under different angles of view and calculate the relative position between the pixels in the image information through different visual imaging principles. At last, people will infer the space position of the target object. Stereo vision is applied valuably in many fields, such as micro position detection and controlling of the operating system, robot navigation and aerial survey, 3D measurement and virtual reality, showing a person with stereo feeling and good viewing effect [1]. In order to respond to the stereo vision research, this paper mainly introduces the stereo vision system and the main production process of 3D TV program. It briefly recommends the stereo television shooting method and error controlling and points out the advantages and disadvantages of various methods and notable problems. Finally, this paper indicates the problems existing in the stereo vision research and its direction of development.

\section{STEREO VISION}

Stereo vision is a feeling of the human eyes when we observe things. Furthermore, the human eyes possess a certain depth perception, and the perception is derived from that the human eyes can extract depth of field in sight. People's stereo vision is established in which the eyes look at an object at the same time and the eye lines of sight cross in one point. The point is called the fixation point, and the point of light is reflected back to the retina from the fixation point.
Both point signal to the brain central of sight for a synthetic and complete object. Not only to see it, but the distance, depth, bump and others between this point and the surrounding objects can tell. Image constituted as this is stereo. This vision is also called stereo vision [2].

The reason why people can form a stereo vision relies mainly on several function of the human eye as follows:

- $\quad$ Binocular parallax: because of spacing (average 6.5 $\mathrm{cm}$ ) between two eyes, so for the same scenery, around the eye position is different, which creates binocular parallax, the left and right eye images are very different.

- Motion parallax: motion parallax is generated by relative movement in observer and scenery. The movement makes the scene size and location of the projection in the retina change, creating depth perception.

- Adaptive adjustment of the eye: the adaptability of the human eye mainly means the active behavior of the eye that adjusting focus. The focal length of the eye can be subtly adjusted through the lens of its internal structure .Change of the focal length allows us to see objects at different distances and different parts of the same scene. In general, the human eye, the minimum focal length is $1.7 \mathrm{~cm}$, there is no upper limit. And the adjustment of the lens is realized through its affiliated muscle contraction and relaxation. Feedback of muscle movement information to the brain contributes to the establishment of the stereo feeling.

- The parallax image fusion in the brain: the binocular image fusion process, the first thing to rely on the same convergence mechanism as the eyes watch the scene, namely the eyes looking at the same point. This mechanism makes both eyes ( the eye distance is certain of one) and the respect in scene on the geometry form a triangle. Through this triangle, we can judge the distance from the observed scene to the human eye. To achieve this mechanism, the human eye muscles need make eyeball movement. Again, muscle activity feeds back to the brain, the parallax images eyes get fusing in the brain. 
In addition to the above several kinds of function, the study suggests that the person's experience and the psychological effect also have an effect on the scene depth perception, such as image of the color difference, the difference of contrast, the scene shadow even the displayer's size and observer's environment. But these factors are negligible in creating stereo feeling relative to the former function.

\section{DEVELOPMENT SitUATION OF STEREO Vision}

The research of stereo vision started in the middle of the 1960s. The 3D scene analysis work was completed by Robert, in the MIT. The work made the past 2D image analysis generalize to 3D scene and marked the birth of stereo vision technology. And in the next following 40 years, it had developed rapidly and became a new subject. Especially in the late 70s, visual calculation theory which was founded by Marr had a great influence on the development of stereo vision. It had formed a complete system from image capture to the final scene of a visual surface reconstruction now. After 40 years of research, the application of stereo vision is increasingly wide in robot vision, aviation mapping, military applications, medical diagnosis and industrial tests [3]. Compared with the traditional 2D, stereo effect of 3D in film and television is the most powerful advantages. Effects of different in essence make 3D movie seems to have an advantage of the world. For the development of stereo television, according to statistics, there are 25 3D TV channels in the world. While, $3 \mathrm{D}$ industry is still in its infancy and not very mature in China, although it develops rapidly, regardless of its early stages of digital film. For domestic stereo television development, its first 3D television channel tested at 19:30 on January 1, 2012; by CCTV, Beijing TV, Shanghai Radio and Television, Tianjin TV, Jiangsu Radio and Television, and Shenzhen Radio and Television. The programs are made by the six agencies respectively and broadcast uniformly by CCTV-4 [4].

\section{STEREO VISION SYSTEM}

The basic principle of stereo vision is to observe the same scene from two (or more) perspective. By calculating the position deviation between image pixels (i.e., parallax) through triangulation principle to obtain the 3D scene information, it can get the perception of images under different angle of viewing. The process is similar to the stereo perception of human visual process. A complete stereo vision system is usually divided into image acquisition, camera calibration, feature extraction, stereo matching, depth determination and interpolation [5].

\section{3D TV PROGRAM PRODUCTION}

Using the $3 \mathrm{D}$ camera or computer, we can get $3 \mathrm{D}$ video signals. Then series of processing can be applied in the left and right signals, such as 3D adjustment, superimposed subtitles, etc. Finally splice the left and the right signals and make them become one that can broadcast in the HD channel. The Fig. 1 followed shows the process clearly.

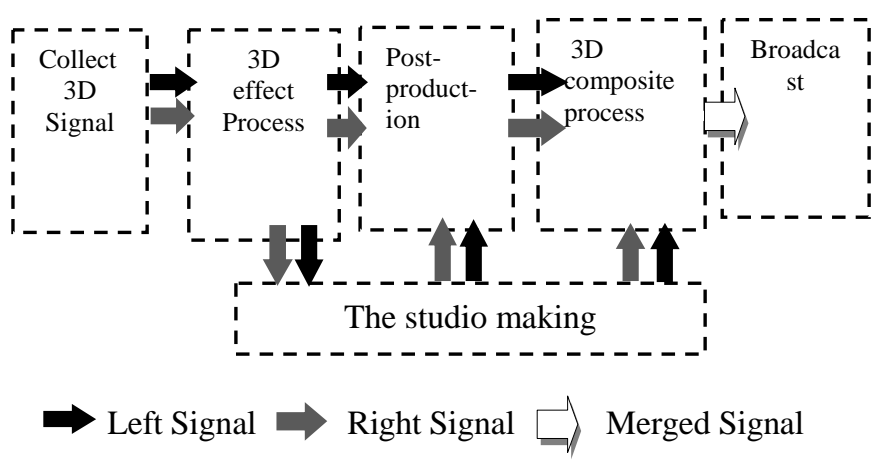

Figure1. Basic Flow of 3D TV Program

\section{A. Convergent method and Parallel method}

Convergent method is one of the 3D TV shooting methods. The optical axis of the left and right lens of the camera tilts inside and intersects at the intersection. Parallel method is another one, whose optical axis of the left and right lens of the camera is parallel in shooting. And parallax of all is negative. The nearer the distance of thing from the camera is, the bigger the negative parallax is. The former one is mainly be adopted.

\section{B. Some 3D TV camera types}

3D TV camera is mainly divided into single machine with single lens and double machine with double lens. One single space of double lens camera can't be changed and this camera can be used in the finite depth range of programs. Double machine with double lens camera can be fixed using horizontal or vertical bracket. Its shooting range is flexible, and it is the main equipment used in the television program production. The method of shooting 3D program can generally be divided into double camera with stereo stents and the integration of 3D camera. Level (tied) double camera is suitable for situation that the main body is far. As the distance between the two cameras' axis is difficult to be close to the space of vertical (spectroscopy) type of double cameras' eyes, this way is not applied to close-up shots. Vertical (spectroscope) type of double camera is suitable for situation that the main body is relatively close. Because of reflected image's reverted image, the picture still need to be adjusted, and the support structure is relatively complex. Therefore, this way is not suitable for long-distance shooting. Shooting occasion of integrated 3D camera: the integration of 3D camera has two lenses; camera unit is installed in horizontal parallel way within a body, and the appearance, weight, volume and operating mode are similar to ordinary camera; but, its advantages are the parallax adjustment function and control of all kinds of error in the design and manufacture. It is applied to low film quality and needed to be taken mobile or to reduce the cost. Integration 3D camera has the relatively small spacing. When it shoots without the prospect of a close shot object lens, stereo vision scene hierarchies is difficult to present. So, generally, when taking 
distant scenery, the level of support with large spacing is needed [6].

\section{Error Controlling methods}

When someone watches 3D TV program, the main reason of the discomfort is due to improper vision, safety control in the filming and production and some parameters over the range of people's tolerance. In order to reduce the feeling of discomfort as far as possible under the premise of maintaining appropriate third dimension, it is necessary to control visual safety during producing 3D program.3D visual safety control includes vision parallax management, error control and vision conflict management. Keeping positive parallax and negative parallax within a certain range in parallax safety management is necessary. If the viewer watches in 3 times distance of $40 \sim 70$ - inch's screen height, positive parallax should be controlled within $50 \mathrm{~mm}$ and negative parallax should be controlled in less than $150 \mathrm{~mm}$ [7].

When the viewer watches for a long time, in order to guarantee comfort, most of the time, the main content of parallax angle should be less than 1 degree, which is equivalent to less than the $3 \%$ of high-definition television in horizontal direction (about 58 pixels) around eyes parallax on screen. The basic method to control parallax in shooting is adjusting the spacing and converging angle of the left and right camera unit.

Image error includes geometric error, electrical error and optical error. Among them, the geometric error can be divided into height error, rotating error, size error, etc..Electrical error can be divided into intensity level difference, color difference, delay difference and signal synchronization inconsistencies of left and right eye image, etc..Optical error is divided into disorder of focus match, zoom matching disorders, focus error and differences between stray light and glare, etc. [8].

The methods of controlling geometric error: firstly, adjust 3D support and pair camera lens to reduce error, then, adopt digital processing method to revise residual error. Digital method can process image real-time or non real-time, but will causes a drop in image quality.

The methods of controlling electrical error: before formal shooting, adjust the camera unit parameters, as far as possible, to keep electrical performance consistency of the camera unit; when shooting, use real-time or non real-time digital processing equipment to correct electrical error.

The methods of controlling optical error: reducing the focusing disorder of matching method is to precisely select matching lens, synchronizing the focus of both lens as far as possible. The method of reducing the focus error is to accurately adjust installation position of camera unit on 3D support, which can make the focus plane of the dual camera unit consistent as much as possible. Glare produced by lens cannot be eliminated and only can be avoided as far as possible when shooting. Ghost caused by reflecting mirror can be reduced or eliminated only by using the high quality mirror.

\section{SUMMARY}

Stereo vision is a crossed-subject that can drive the development of film and television industry, promote the scientific research ability and gain more valuable information. Along with the advance of science and technology, the integrated application of various techniques in study of stereo vision will elevate the manufacturing flow of stereo vision and broadcast effect and also can promote human civilization and progress.

\section{ACKNOWLEDGMENT}

This paper is supported by the national sci-tech support plan project Key Technology Research and Application of Stereo Vision Content Services whose number is 2012BAH37F03. Thanks for the work of the refereeing.

\section{REFERENCES}

[1] Suya You, Guang Xu, Present situation and progress in the study of stereo vision, Journal of Chinese image and graphics, 2 (1), 1997.

[2] Dawei Liu , Shengyong Chen , Xiaoyan Wang, Research on 3D Reconstruction Method for Stereo Vision,College of Information Engineering Zhejiang University of Technology, May 2011.

[3] Hua Pan, Ge Guo, Research progress of stereo vision, Computer Measurement and Control, 2004.12 (12).

[4] Bin Kong, latest development and research of 3D TV, CCBN Special Issue, 2012, 3.

[5] Zhen He,Qiongyan Li,Jiangming Kan, Research of Camera Calibration Based on Stereo Vision,Science Technology Innovation Herald,2012 NO.15.

[6] Xiaobo Lai, Several key Problems and Technology research of Robot binocular Stereo Vision Theory, University of Zhejiang ,2010 Ph.D. Thesis.

[7] Yuqing Cheng,The latest technology research of Subjective and Objective evaluation of 3D TV video quality, Journal of Communication University of China, 2012.3.

[8] Qingding Ding, Jilin Liu ,Research on Several Problems for the Practical Use of Stereo Vision, College of Information Engineering Zhejiang University of Technology, June 2012. 